\title{
Quercetin reduces oxidative stress and inhibits activation of c-Jun N-terminal kinase/activator protein-1 signaling in an experimental mouse model of abdominal aortic aneurysm
}

\author{
LIAN WANG $^{1}$, XIAOFENG CHENG ${ }^{2}, \mathrm{HAO} \mathrm{LI}^{2}, \mathrm{FANG} \mathrm{QIU}^{3}, \mathrm{NAN} \mathrm{YANG}^{2}, \mathrm{BO} \mathrm{WANG}^{2}$, \\ HUCHEN LU ${ }^{4}$, HAIWEI WU ${ }^{2}$, YI SHEN ${ }^{2}$, YANQING WANG ${ }^{5}$ and HUA JING ${ }^{2}$
}

\begin{abstract}
${ }^{1}$ Department of Thoracic Surgery, Second Affiliated Hospital, Zhejiang University School of Medicine, Hangzhou, Zhejiang 310009; ${ }^{2}$ Department of Cardiothoracic Surgery, Jinling Hospital, Clinical Medicine School of Nanjing University;

${ }^{3}$ D.A. Diagnostic Laboratory; ${ }^{4}$ Department of Neurological Surgery, Jinling Hospital, Clinical Medicine School of Nanjing University, Nanjing, Jiangsu 210009; ${ }^{5}$ Department of Cardiology,

The 81 st Hospital of PLA, Nanjing, Jiangsu 210002, P.R. China
\end{abstract}

Received May 23, 2013; Accepted November 26, 2013

DOI: $10.3892 / \mathrm{mmr} .2013 .1846$

\begin{abstract}
Oxidative stress is becoming increasingly linked to the pathogenesis of abdominal aortic aneurysms (AAAs). The antioxidant activity of flavonoids has attracted attention for their possible role in the prevention of cardiovascular diseases. The purpose of this study was to determine whether an antioxidant mechanism is involved in the aneurysm formation inhibitory effect afforded by quercetin. Male C57/BL6 mice received quercetin continuously from 2 weeks prior to and 6 weeks following the AAA induction with extraluminal $\mathrm{CaCl}_{2}$. Quercetin treatment decreased AAA incidence and inhibited the reactive oxygen species generation, nitrotyrosine formation and lipid peroxidation production in the aortic tissue during AAA development. In addition, quercetin-treated mice exhibited significantly lower expression of the $\mathrm{p} 47 \mathrm{phox}$ subunit of nicotinamide adenine dinucleotide phosphate oxidase and inducible nitric oxide synthase, as well as coordinated downregulation of manganese-superoxide dismutase activities and glutathione peroxidase (GPx)-1 and GPx-3 expression. Quercetin also blunted the expression of c-Jun N-terminal kinase (JNK) and phospho-JNK and, in addition, diminished activation of the activator protein (AP)-1 transcription factor. Gelatin zymography showed that quercetin eliminated matrix
\end{abstract}

Correspondence to: Dr Yanqing Wang, Department of Cardiology, The 81st Hospital of PLA, 34-34 Yanggongjing, Nanjing, Jiangsu 210002, P.R. China

E-mail: yanqing81c@gmail.com

Dr Yi Shen, Department of Cardiothoracic Surgery, Jinling Hospital, Clinical Medicine School of Nanjing University, 305 East Zhongshan Road, Nanjing, Jiangsu 210009, China

E-mail: drshenyi@hotmail.com

Key words: quercetin, abdominal aortic aneurysm, oxidative stress, nicotinamide adenine dinucleotide phosphate oxidase, c-Jun $\mathrm{N}$-terminal kinase, activator protein-1 metalloproteinase (MMP)-2 and MMP-9 activation during AAA formation. In conclusion, the inhibitory effects of quercetin on oxidative stress and MMP activation, through modulation of JNK/AP-1 signaling, may partly account for its benefit in $\mathrm{CaCl}_{2}$-induced AAA.

\section{Introduction}

An abdominal aortic aneurysm (AAA) is a localized, permanent dilatation of the aorta that affects $\sim 8 \%$ of males $>65$ years-old (1). At present, elective surgery is the major therapeutic option for AAA, however, this is not applicable to small aneurysms despite the reported growth rate of small aneurysms ranging between 1.5 and $3 \mathrm{~mm}$ per year, which leads to a higher risk of rupture (2). With increased knowledge of aneurysm pathophysiology, it is possible that aneurysm growth may be retarded with medical therapy.

The role of inflammation in the pathogenesis of AAA is well established. Infiltrating inflammatory cells enter the aorta, release cytokines and proteases, inducing apoptosis of vascular smooth muscle cells and ultimately, lead to destruction of the vascular wall (1). Moreover, the inflammatory microenvironment generates a large quantity of oxidant species, largely through upregulation of nicotinamide adenine dinucleotide phosphate (NADPH) oxidase in vascular cells (3). Furthermore, emerging evidence indicates that oxidative stress within the aortic wall is closely involved in the pathogenesis of AAA. Oxidative stress facilitates leukocyte recruitment into the vasculature by modulating adhesion molecules and chemotactic cytokines (4). In addition, reactive oxygen species (ROS) may alter the balance between destruction and regeneration of the aortic wall by enhancing matrix proteolysis through upregulation of matrix metalloproteinases (MMPs) (5). MMPs are the predominant extracellular proteinases that participate in the degradation process of structural proteins (1). Although human data remains limited, several studies indicate that antioxidant therapy may be effective in experimental AAA models (6-8). 
Quercetin (3,5,7,3'4'-Pentahydroxy flavon), a typical member of the flavonoid family, is one of the most widely recognized dietary polyphenolic compounds. It is ubiquitously present in foods and is claimed to exert beneficial effects on vascular disease (9), which has been largely associated with its antioxidant and anti-inflammatory properties. Within the flavonoid family, quercetin is proven to be the most potent scavenger of free radicals (10). There is evidence that quercetin reduces low-density lipoprotein oxidation (11) and prevents the development of atherosclerotic lesions (12), in which oxidative stress is assumed to have a pivotal role. Although atherosclerosis and AAA are separate diseases, they have certain similar pathological characteristics, including inflammation and proteolysis (13). It is also reported that quercetin in vitro inhibits the production of $\mathrm{O}_{2}{ }^{--}$in the rat aorta and decreases protein expression of the NADPH oxidase subunit, p47phox $(14,15)$. A previous study from our research group indicated that quercetin treatment inhibits inflammation and prevents $\mathrm{CaCl}_{2}$-induced aneurysmal dilation in a mouse AAA model (16). The present study was designed to test the hypothesis that an antioxidative mechanism is also involved in the protection afforded by quercetin.

\section{Materials and methods}

Pharmacological treatments. Quercetin was purchased from Sigma-Aldrich (Q4951; Shanghai, China). Drug solutions were prepared by suspending the compound in $0.5 \%$ carboxymethyl cellulose sodium. Animals were gavaged daily with $0.1 \mathrm{ml}$ solution of quercetin $(60 \mathrm{mg} / \mathrm{kg})$ or vehicle alone, which began 2 weeks prior to AAA induction and continued for 8 weeks. The dose regimen for quercetin was based on previous studies demonstrating beneficial effects of the drug in mouse models of aortic atherosclerosis (12).

Animal groups and the AAA model. A total of 60 male C57BL/6 wild-type mice (age, 6-7 weeks) were obtained from Vital River Laboratory Animal Technology (Beijing, China). All animals were treated and cared for in accordance with the Guide for the Care and Use of Laboratory Animals (National Institutes of Health, Washington DC, 1996) and the experimental protocols were approved by the Animal Care and Use Committee (Nanjing University, Nanjing, China). The mice were randomly assigned to one of four groups ( $n=15$ in each group): Vehicle treatment plus sham operation control (VC), vehicle treatment plus AAA (VA), quercetin treatment plus AAA (QA) and quercetin treatment plus sham operation control (QC). AAA was induced in the infrarenal abdominal aorta (age, 8 weeks) by periaortic application of $\mathrm{CaCl}_{2}$, as previously described (16). $\mathrm{NaCl}(0.9 \%)$ was substituted for $\mathrm{CaCl}_{2}$ in sham operation animals. Six weeks later, the mice were laparotomied and the aortic diameters (ADs) were measured; the abdominal incision was carried upwards as a thoracoabdominal incision, the animals were then sacrificed by left-heart injection of potassium chloride and the aortic tissues were collected. An aneurysm was defined as an increase in the $\mathrm{AD}$ of $>50 \%$ of the original $\mathrm{AD}$.

In vivo hemodynamic measurements. A computerized, non-invasive tail-cuff system with a four-channel mouse plat- form (BP-2000; Visitech Systems, Inc., Apex, NC, USA) was used to measure blood pressure and heart rate. To train mice, daily measurements were performed for five consecutive days prior to the actual recorded measurements. Hemodynamic parameters were measured one day pre- and 6 weeks post-AAA induction. The first 10 of 30 values recorded at each session were disregarded and the remaining 20 values were averaged and used for analysis, according to the manufacturer's instructions.

ROS analysis, lipid peroxidation determination and manganese-superoxide dismutase (Mn-SOD) activity assay. Dihydroethidium (DHE) oxidative fluorescence dye was used to evaluate in situ production of ROS (17). DHE stock solution was prepared by dissolving DHE (D7008; Sigma-Aldrich) in dimethylsulfoxide at a concentration of $5 \mathrm{mM}$. The stock solution was stored in the dark and diluted in phosphate-buffered saline (PBS) to a final concentration of $5 \mu \mathrm{M}$ immediately prior to use. The abdominal aorta was harvested and the aortic segment $(10 \mathrm{~mm})$ was embedded in Tissue-Tek OCT compound (Sakura Finetech Japan, Tokyo, Japan) and snap-frozen. DHE working solution $(200 \mu \mathrm{l})$ was topically applied to the aortic sections and the slides were subsequently incubated at $37^{\circ} \mathrm{C}$ in the dark for $30 \mathrm{~min}$. Excess DHE was rinsed off twice with PBS and the images were immediately captured with a fluorescent microscope (BX51; Olympus, Tokyo, Japan) at excitation and emission wavelengths of 520 and $610 \mathrm{~nm}$, respectively.

A portion of the snap-frozen aortic tissue ( $\mathrm{n}=5$ per group) was crushed in a prechilled mortar and resuspended in PBS at a concentration of $50 \mathrm{mg} / \mathrm{ml}$. The homogenate was centrifuged at $10,000 \mathrm{x}$ g for $10 \mathrm{~min}$ at $4^{\circ} \mathrm{C}$ to collect the supernatant. The lipid peroxidation product, malondialdehyde (MDA), was assessed using the thiobarbituric acid reactive substances (TBARS) assay kit (A003; Jiancheng Bioengineering, Shanghai, China). Briefly, $100 \mu 1$ supernatant was added to $100 \mu 1$ sodium dodecyl sulfate (SDS) lysis solution and mixed thoroughly. Following the addition of $250 \mu \mathrm{l}$ thiobarbituric acid (TBA) reagent, samples were incubated at $95^{\circ} \mathrm{C}$ for $1 \mathrm{~h}$ and centrifuged at $1,500 \mathrm{x} \mathrm{g}$ at room temperature for $15 \mathrm{~min}$. The absorbance of each supernatant was measured at $532 \mathrm{~nm}$ using a spectrophotometer (BioPhotometer; Eppendorf, Hamburg, Germany). Values of TBARS are expressed as nmol equivalents of MDA per mg protein. Mn-SOD activity was measured using an assay kit (A001-2; Jiancheng Bioengineering) according to the manufacturer's instructions. Assay conditions were $65 \mu \mathrm{mol}$ phosphate buffer (pH 7.8), $1 \mu$ mol hydrochloric hydroxylamine, $0.75 \mu \mathrm{mol}$ xanthine and $2.3 \times 10^{-3} \mathrm{IU}$ xanthine dismutase. The supernatant (50 $\mu \mathrm{l}$ ) was incubated in the system for $40 \mathrm{~min}$ at $37^{\circ} \mathrm{C}$ and terminated with $2 \mathrm{ml} 3.3 \mathrm{~g} / \mathrm{l} \mathrm{p}$-aminobenzene sulfonic acid and $10 \mathrm{~g} / \mathrm{l}$ naphthylamine. For inhibition of CuZn-SOD activity, the assay was conducted in the presence of $10 \mathrm{~mm} \mathrm{KCN}$ following preincubation for $30 \mathrm{~min}$. The supernatant was transferred to a microplate (Eppendorf) for determination of the absorbance at $550 \mathrm{~nm}$ and 1 unit SOD was defined as the quantity of enzyme required to produce $50 \%$ dismutation of superoxide radical. Mn-SOD activity was calculated by subtraction of CuZn-SOD activity from total SOD activity. The standard curves were created as described in the manufacturer's instructions. Images were assessed by Image J 1.44 software (National Institute of Health, Bethesda, MD, USA). 
Histological analysis. The infrarenal abdominal aorta $(\mathrm{n}=5$ per group) was dissected and fixed in $10 \%$ neutral-buffered formalin. Specimens were dehydrated through graded ethanols, embedded in paraffin and sliced into 4-6- $\mu \mathrm{m}$ sections. Immunohistochemical staining with a rabbit polyclonal anti-nitrotyrosine antibody (1:500; 06-284; Millipore, Temecula, CA, USA) was used as an indicator of peroxynitrite formation (18). Briefly, the slides were incubated in 3\% hydrogen peroxide for $5 \mathrm{~min}$ to quench endogenous peroxidase activity and were then incubated with primary antibody overnight at $4^{\circ} \mathrm{C}$. Subsequently, slides were washed with PBS and incubated $\left(15 \mathrm{~min} ; 37^{\circ} \mathrm{C}\right)$ with peroxidase-conjugated goat anti-rabbit IgG (AP132P; Millipore). Finally, the slides were incubated with diaminobenzidine and counterstained with hematoxylin.

Reverse transcription-polymerase chain reaction (RT-PCR). RT-PCR was used to define the expression of glutathione peroxidase (GPx)-1, GPx-3, inducible nitric oxide synthase (iNOS) and p47phox NADPH oxidase mRNA. Total RNA was prepared with the TRIzol total RNA extraction kit (SK1321; Sangon Biotech, Shanghai, China). Primer sequences were as follows: Forward: 5'-ACC CCA AGT ACA TCA TTT GGT C-3' and reverse: 5'-GCA GGG TTT CTA TGT CAG GTT C-3' for GPx-1; forward: 5'-ATC TAC GAG TAT GGA GCC CTC A-3' and reverse: 5'-GGC CCA AGT TCT TCT TGT AGT G-3' for GPx-3; forward: 5'-CTT TGA CGC TCG GAA CTG TAG-3' and reverse: 5'-AAC TCC AAG GTG GCA GCA T-3' for iNOS; forward: 5'-CCC ATC ATC CTT CAG ACC TAT C-3' and reverse: 5'-AAC CTC GCT TTG TCT TCA TCT G-3' for p47 NADPH oxidase; and forward: 5'-AGG CCG GTG CTG AGT ATG TC-3' and reverse: 5'-TGC CTG CTT CAC CAC CTT CT-3' for GAPDH. Reverse transcription was performed with oligo-dT primers and the AMV First Strand cDNA Synthesis kit (SK2029; Sangon Biotech), according to the manufacturer's instructions. The resultant cDNA was amplified by Taq DNA polymerase (SK2442; Sangon Biotech) in an Access RT-PCR System (Promega Corp., Madison, WI, USA). GAPDH mRNA was also amplified to serve as an internal control. The resultant PCR products were detected using an MSF-300G Scanner (Microtek Lab, Carson, CA, USA) and expressed as the ratio to GAPDH.

Western blotting. Total protein was extracted from the supernatants of tissue homogenate with T-PER tissue protein extraction reagent (Pierce Biotechnology Inc., Rockford, IL, USA) and stored at $-80^{\circ} \mathrm{C}$. Equal quantities $(30 \mu \mathrm{g})$ of total protein were separated on $10 \%$ polyacrylamide gels and transferred to nitrocellulose membranes using a semidry transfer cell (\#164-5052; Bio-Rad, Hercules, CA, USA) at $10 \mathrm{~V}$ for $40 \mathrm{~min}$. The membranes were blocked for $60 \mathrm{~min}$ with 5\% nonfat milk in Tris-buffered saline with Tween-20 (TBST) and subsequently washed. Primary antibodies for p47phox NADPH oxidase (sc-14015), c-Jun N-terminal kinase (JNK; sc-571) and phosphorylated JNK (sc-6254) (all Santa Cruz Biotechnology, Santa Cruz, CA, USA) were added at a 1:500 dilution and incubated overnight at $4^{\circ} \mathrm{C}$. Additionally, all blots were incubated with the anti- $\beta$-actin antibody (1:5,000; 4970; Cell Signaling Technology, Beverly, MA, USA) to confirm protein loading levels. Membranes were washed with TBST, incubated with horseradish peroxidase-conjugated species-appropriate secondary antibodies (Santa Cruz Biotechnology) for $1 \mathrm{~h}$ at room temperature and developed using an enhanced chemiluminescence kit (Pierce, Rockford, IL, USA). Quantification of images was performed by scanning densitometry with Image J 1.44.

Electrophoretic mobility shift assay (EMSA). Nuclear protein lysates were harvested using NE-PER nuclear and cytoplasmic extraction reagents (Pierce Biotechnology, Inc.) according to the manufacturer's instructions. Activator protein (AP)-1 DNA-binding activities were analyzed using Gel Shift Assay systems (Promega Corporation, Madison, WI, USA), according to the instructions previously described (16). Briefly, the AP-1 consensus oligonucleotide probe (5'-CGC TTG ATG AGT CAG CCG GAA-3') was end-labeled with $\left[\gamma^{32} \mathrm{P}\right]-\mathrm{ATP}$ (Furui Biotech, Beijing, China). The extracted nuclear proteins $(10 \mu \mathrm{g})$ were incubated for $20 \mathrm{~min}$ at $37^{\circ} \mathrm{C}$ with the ${ }^{32} \mathrm{P}$-labeled oligonucleotide $(0.30 \mathrm{pmol})$ in a binding buffer. Reaction products were then separated in a $4 \%$ polyacrylamide gel, followed by autoradiography. The reactive bands were quantified as described in western blot analysis.

Gelatin zymography. Protein extracts $(10 \mu \mathrm{g})$ were mixed with SDS buffer and separated by electrophoresis on $10 \%$ SDS-polyacrylamide gels containing $1.0 \%$ gelatin. Following electrophoresis, the gels were renatured in renaturing buffer (LC2670; Invitrogen Life Technologies, Carlsbad, CA, USA) and incubated with developing buffer (LC2671; Invitrogen Life Technologies) for $30 \mathrm{~min}$ at room temperature. Subsequently, the gel was incubated in fresh developing buffer overnight at $37^{\circ} \mathrm{C}$. The gel was stained with $0.5 \%$ Coomassie blue R-250 for $30 \mathrm{~min}$ and destained with destaining solution containing $10 \%$ acetic acid and $40 \%$ methanol. The relative molecular weight of each band was determined using protein standards (Pierce Biotechnology, Inc.). Areas of protease activity appeared as unstained bands against a blue background. Images were assessed by Image $\mathrm{J} 1.44$.

Statistical Analysis. All values are expressed as the mean \pm standard deviation. Statistical analyses were performed with SPSS for Windows version 17.0 (SPSS, Inc., Chicago, IL, USA). Within-group comparisons of hemodynamic parameters at various intervals were performed using paired Student's t tests. Between-group comparisons were performed using the Fisher's exact test or analysis of variance. $\mathrm{P}<0.05$ was considered to indicate a statistically significant difference.

\section{Results}

$A A A$ incidence. No significant difference was found in AD at the time of surgery among the groups (data not shown). Six weeks later, the VA mice showed a marked increase in AD following $\mathrm{CaCl}_{2}$ treatment with 10/15 (66.7\%) developing aneurysms. Only 3/15 (20\%) of the aortas became aneurysmal in QA mice; this difference in aneurysm incidence was considered to be highly significant $(\mathrm{P}<0.05)$. No aneurysm formation was observed in the VC or QC mice. Quercetin treatment had no effect on mean arterial pressure or heart rate when measured pre- and post-surgery (Table I). 
Table I. Heart rate and mean arterial pressure prior to and following $\mathrm{NaCl} / \mathrm{CaCl}_{2}$ treatment.

\begin{tabular}{|c|c|c|c|c|c|c|}
\hline \multirow{2}{*}{$\begin{array}{l}\text { Group } \\
(n=15)\end{array}$} & \multicolumn{2}{|c|}{2 weeks prior to surgery } & \multicolumn{2}{|c|}{ Surgery } & \multicolumn{2}{|c|}{6 weeks post-surgery } \\
\hline & $\mathrm{HR}, \mathrm{bpm}$ & MAP, $\mathrm{mmHg}$ & $\mathrm{HR}, \mathrm{bpm}$ & MAP, mmHg & $\mathrm{HR}, \mathrm{bpm}$ & MAP, $\mathrm{mmHg}$ \\
\hline $\mathrm{VC}$ & $630 \pm 30$ & $83 \pm 8$ & $626 \pm 34$ & $85 \pm 6$ & $633 \pm 27$ & $85 \pm 6$ \\
\hline VA & $628 \pm 28$ & $85 \pm 7$ & $632 \pm 29$ & $82 \pm 5$ & $631 \pm 35$ & $86 \pm 9$ \\
\hline QA & $631 \pm 33$ & $84 \pm 6$ & $629 \pm 33$ & $84 \pm 8$ & $627 \pm 34$ & $83 \pm 5$ \\
\hline QC & $634 \pm 31$ & $84 \pm 9$ & $633 \pm 34$ & $85 \pm 5$ & $630 \pm 32$ & $85 \pm 8$ \\
\hline
\end{tabular}

Heart rate and mean arterial pressure were recorded using the tail-cuff system. Measurements are expressed as the mean \pm standard deviation. AAA, abdominal aortic aneurysm; VC, vehicle treatment plus sham operation control; VA, vehicle treatment plus AAA; QA, quercetin treatment plus AAA; QC, quercetin treatment plus sham operation control.

Table II. Effect of quercetin on MDA levels and Mn-SOD activities following $\mathrm{NaCl} / \mathrm{CaCl}_{2}$ incubation.

\begin{tabular}{lcc}
\hline Group & $\begin{array}{c}\text { TBARS } \\
(\mathrm{nm} \text { MDA/mg protein })\end{array}$ & $\begin{array}{c}\text { Mn-SOD activities } \\
\text { (U/mg protein) }\end{array}$ \\
\hline VC & $0.075 \pm 0.027$ & $2.8 \pm 1.5$ \\
VA & $0.342 \pm 0.035^{\mathrm{a}}$ & $28.2 \pm 5.4^{\mathrm{a}}$ \\
QA & $0.231 \pm 0.029^{\mathrm{b}}$ & $15.5 \pm 4.9^{\mathrm{b}}$ \\
QC & $0.069 \pm 0.015$ & $2.5 \pm 1.8$ \\
\hline
\end{tabular}

Values are presented as the mean \pm standard deviation with $n=5$ per group. ${ }^{\mathrm{a}} \mathrm{P}<0.05 \mathrm{vs}$. VC; ${ }^{\mathrm{b}} \mathrm{P}<0.05$ vs. VA. AAA, abdominal aortic aneurysm; MDA, malondialdehyde; Mn-SOD, manganese-superoxide dismutase; TBARS, thiobarbituric acid reactive substances (assay kit); VC, vehicle treatment plus sham operation control; VA, vehicle treatment plus AAA; QA, quercetin treatment plus AAA; QC, quercetin treatment plus sham operation control.

ROS generation, nitrotyrosine formation and lipid peroxidation production. To evaluate the effect of quercetin on ROS generation, aortic sections were exposed to DHE, which is transformed to the highly fluorescent molecule, oxyethidium, in the presence of superoxide (17). As shown in Fig. 1A, ROS production was extremely low in aortas from $\mathrm{VC}$ and QC mice. At 6 weeks post-AAA induction in VA mice, oxyethidium fluorescence was higher, being significantly enhanced throughout the vascular wall (Fig. 1A and B). However, it was attenuated in QA mice, indicating decreased ROS production due to quercetin treatment.

As increased production of ROS may lead to further peroxynitrite accumulation, which induces protein damage by formation of nitrotyrosine (18), immunohistochemistry was performed with a polyclonal antibody against nitrotyrosine in aortic cross sections. Staining appeared only weakly in the aorta of VC and QC animals, however, VA mice revealed marked brown nitrotyrosine staining in the aortic wall. By contrast, a decreased immunoreactivity was observed in QA mice (Fig. 1A and C). Similar observations were noted for lipid peroxidation production (MDA) levels (TBARS) in aortic tissues. The TBARS concentration in QA mice was found to be significantly lower than that in the VA mice (Table II).
Endogenous vascular antioxidant defense systems. Increased levels of Mn-SOD activity were observed at AAA regions of the VA mice, while quercetin significantly decreased its activity in QA mice (Table II). In addition, quercetin caused a relative decrease in mRNA expression of GPx-1 and GPx-3, which are involved in antioxidative status (Fig. 2).

Expression of iNOS and p47phox NADPH oxidase. Expression of iNOS and the NADPH oxidase subunit, p47phox, was also examined. Compared with the VA group, QA mice showed relative decreases in iNOS and p47phox mRNA levels (Fig. 2) and this result was confirmed by western blot analysis of p47phox (Fig. 3A and B).

JNK/AP-1 signaling pathway and enzymatic activities of $M M P s$. Since quercetin reduced oxidative stress, the specific contribution of quercetin to the regulation of AP-1 activation in experimental AAA was examined. Levels of AP-1 DNA binding activity in QA mice, determined by EMSA, were significantly inhibited by quercetin when compared with controls in the VA group (Fig. 4A and B). Western blotting showed that phosphorylated-JNK was significantly upregulated in VA mice and downregulated following treatment with quercetin. In addition, QA animals had significantly less total JNK than VA controls (Fig. 3A and B).

Gelatin zymography revealed that MMP-2 and -9 activities were elevated in VA mice, however, quercetin treatment resulted in a marked decrease in MMP-2 and -9 activities (Fig. 5).

\section{Discussion}

The $\mathrm{CaCl}_{2}$-induced AAA model has been widely employed to gain further understanding of the mechanisms involved in aneurysm development, in order to identify potential novel medical treatments (19). As the results of this study showed, the development of $\mathrm{CaCl}_{2}$-induced AAA in mice was accompanied by elevated aortic ROS levels, increased nitrotyrosine formation and lipid peroxidation products, indicating an enhancement in overall oxidative stress. Previous studies have demonstrated that markers of oxidative damage are present in human (20) and animal (21) aneurysmal lesions. However, these oxidative stress markers were significantly inhibited by 

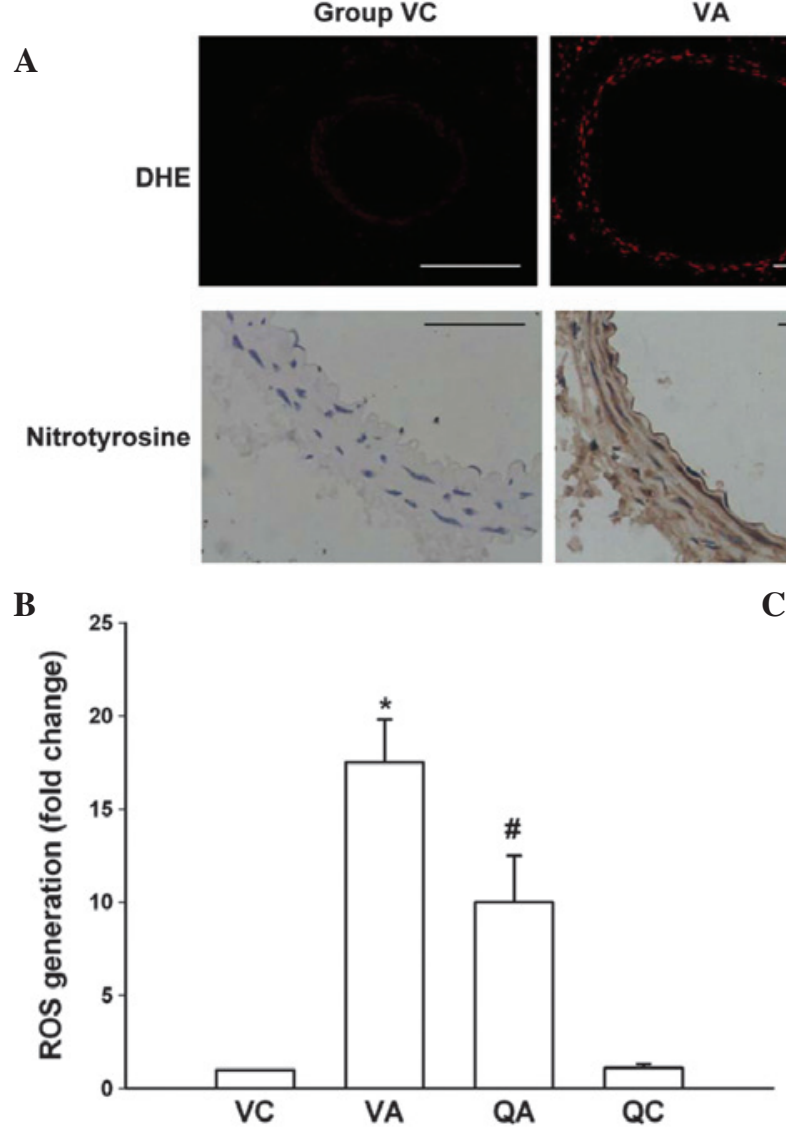

$\mathbf{C}$
VA
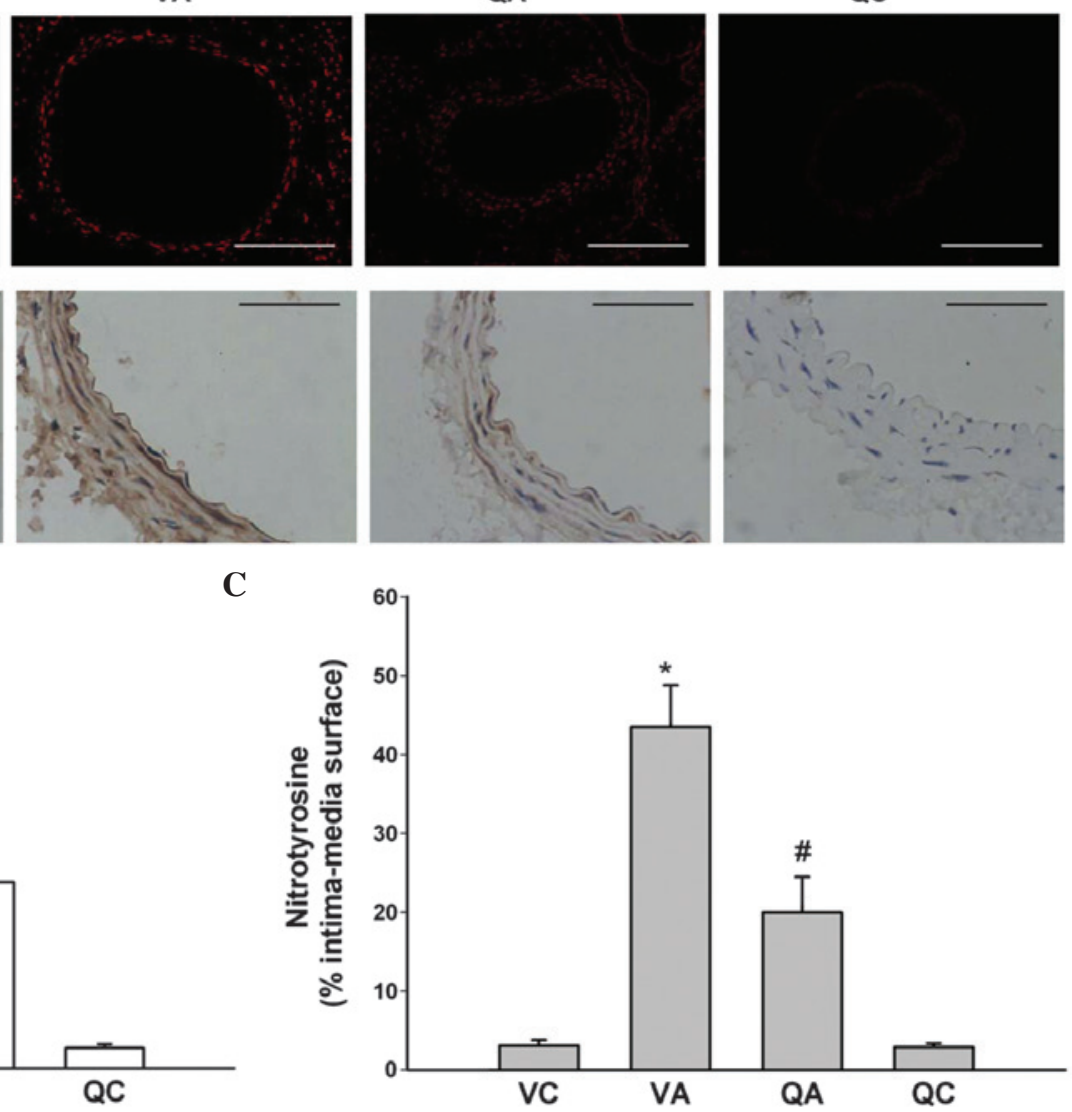

Figure 1. Effect of quercetin on ROS generation and nitrotyrosine formation in the aortic wall. (A) DHE fluorescence imaging and immunohistochemical staining for nitrotyrosine in the mouse aortic wall (details in Materials and methods). Scale bars are 500 and $50 \mu \mathrm{m}$, respectively. (B) Densitometric analysis of DHE fluorescence relative to VC mice. (C) Positively stained areas in the aortic section of each group. Values are presented as the mean \pm standard deviation with $\mathrm{n}=5$ per group. ${ }^{*} \mathrm{P}<0.05$, vs. VC; ${ }^{\prime} \mathrm{P}<0.05$, vs. VA. ROS, reactive oxygen species; DHE, dihydroethidium; AAA, abdominal aortic aneurysm; VC, vehicle treatment plus sham operation control; VA, vehicle treatment plus AAA; QA, quercetin treatment plus AAA; QC, quercetin treatment plus sham operation control.
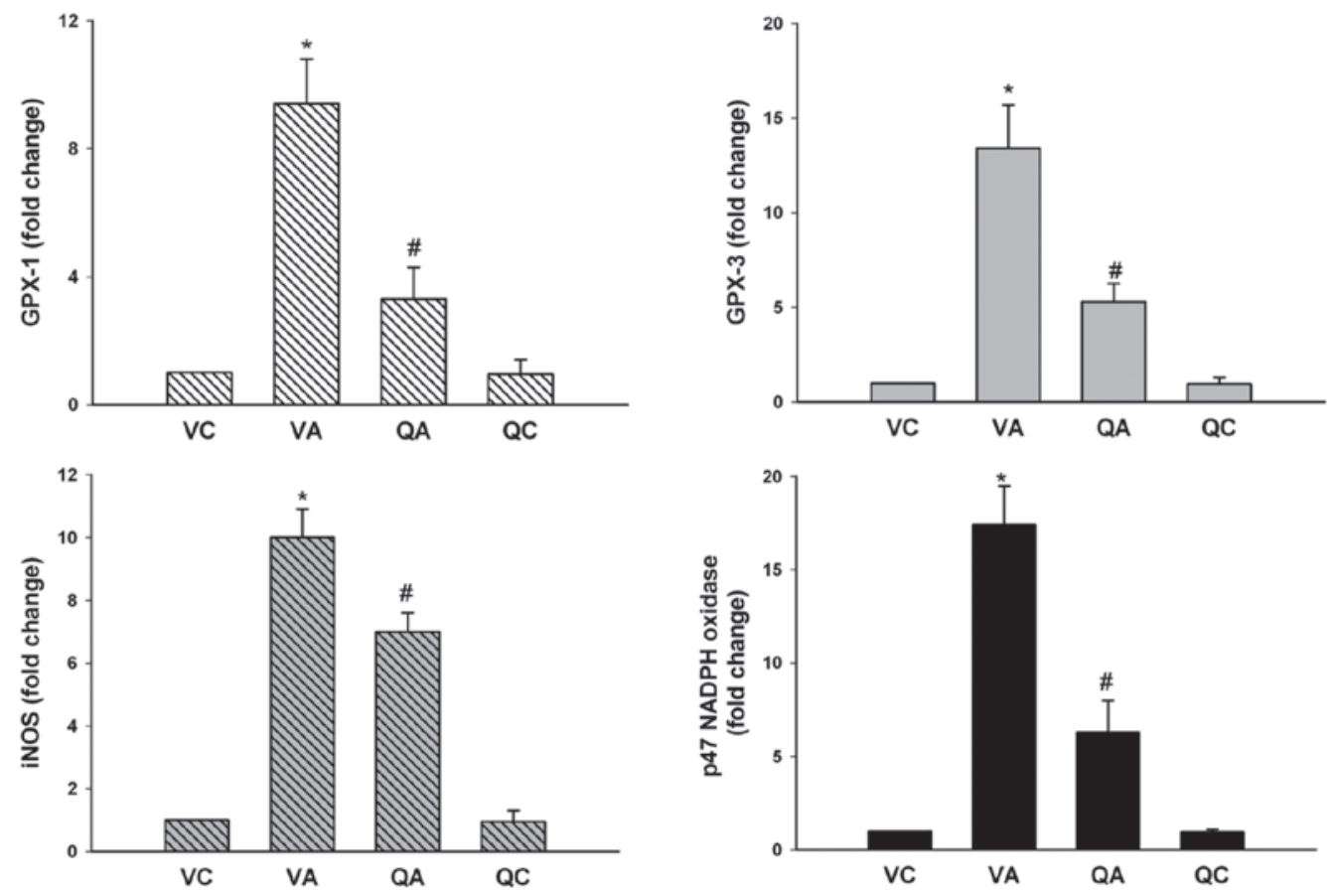

Figure 2. mRNA expression of GPx-1, GPx-3, iNOS and p47 NADPH oxidase. Data are expressed as fold changes compared with values of VC animals (n=5 in each group). "P $<0.05$, vs. VC; ${ }^{*} \mathrm{P}<0.05$, vs. VA. AAA, abdominal aortic aneurysm; iNOS, inducible nitric oxide synthase; Gx, glutathione peroxidase; NADPH, nicotinamide adenine dinucleotide phosphate; VC, vehicle treatment plus sham operation control; VA, vehicle treatment plus AAA; QA, quercetin treatment plus AAA; QC, quercetin treatment plus sham operation control. 
A

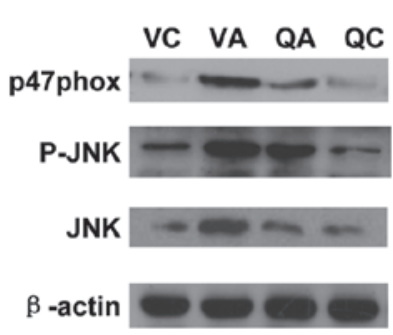

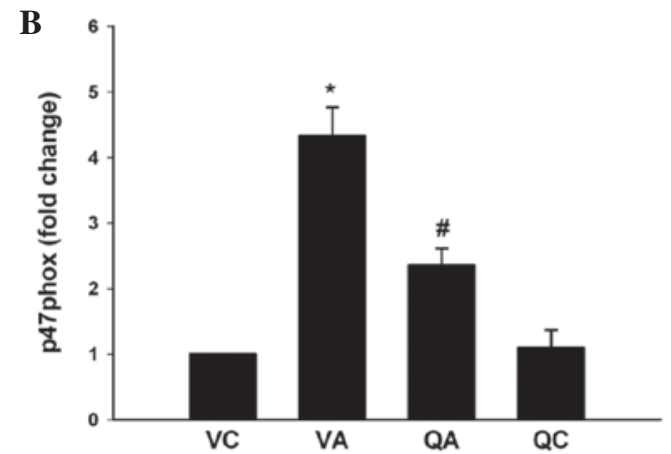

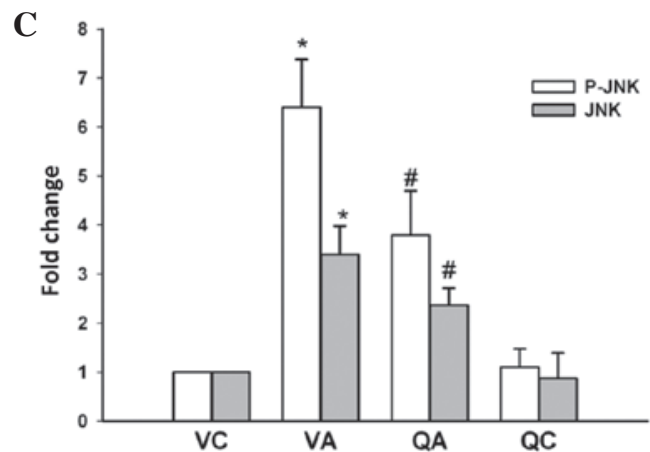

Figure 3. Expression of p47phox NADPH oxidase, phosphorylated JNK and total JNK (A). Data are presented as the mean \pm standard deviation with $\mathrm{n}=5$ per group and expressed as fold changes compared with values in the $\mathrm{VC}$ animals (B). " $\mathrm{P}<0.05$, vs. VC; ${ }^{\text {" }} \mathrm{P}<0.05$, vs. VA. AAA, abdominal aortic aneurysm; JNK, c-Jun N-terminal kinase; NADPH, nicotinamide adenine dinucleotide phosphate; VC, vehicle treatment plus sham operation control; VA, vehicle treatment plus AAA; QA, quercetin treatment plus AAA; QC, quercetin treatment plus sham operation control.

A

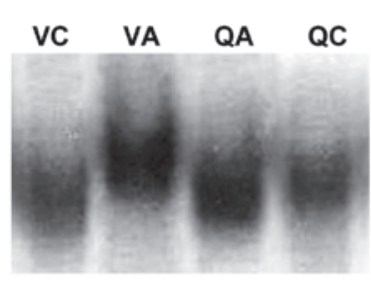

B

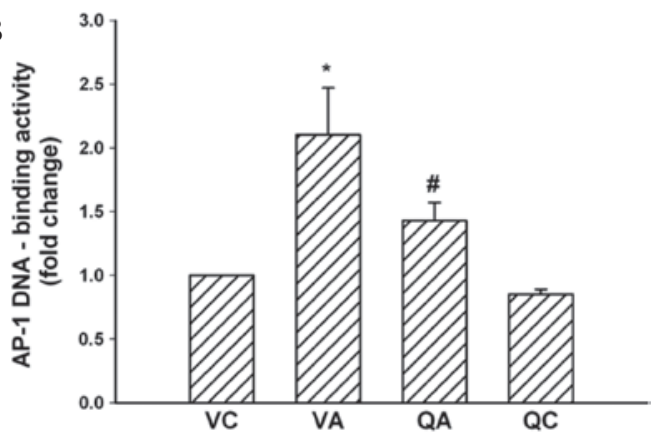

Figure 4. EMSA for AP-1 binding sites. (A) Representative results of EMSA for AP-1 binding sites 6 weeks following $\mathrm{NaCl} / \mathrm{CaCl}_{2}$ incubation. (B) Quantification of the hybridization shown in panel (A) by densitometric analysis. Signals from animals in the VC group were arbitrarily provided as $100 \%$; those from other animals were expressed as the percentage of values in the VC animals. $n=5$ per group. ${ }^{*} \mathrm{P}<0.05$, vs. VC; ${ }^{\text {}} \mathrm{P}<0.05$, vs. VA. EMSA, electrophoretic mobility shift assay; AP-1, activator protein; AAA, abdominal aortic aneurysm; VC, vehicle treatment plus sham operation control; VA, vehicle treatment plus AAA; QA, quercetin treatment plus AAA; QC, quercetin treatment plus sham operation control.

A

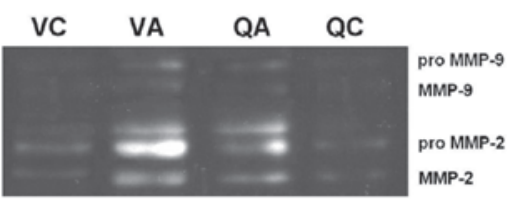

B

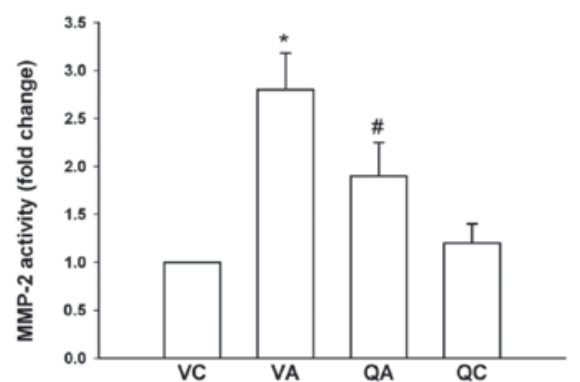

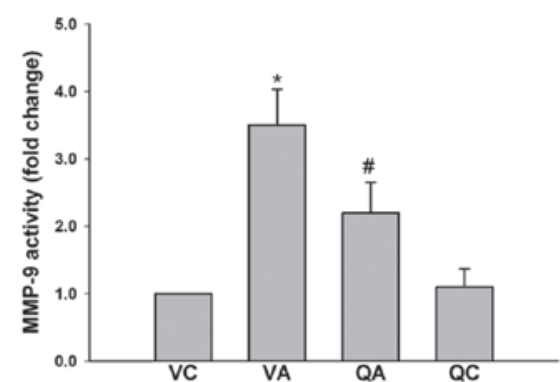

Figure 5. Gelatin zymography analysis of MMP-2 and -9 activities. (A) Representative gelatin zymography at 6 weeks following $\mathrm{NaCl} / \mathrm{CaCl}{ }_{2}$ incubation. (B) Calculated relative density (percentage of VC) of gelatin zymography showed differences in MMP activities between groups ( $\mathrm{n}=5$ per group). " $\mathrm{P}<0.05$, vs. VC; ${ }^{\prime} \mathrm{P}<0.05$, vs. VA. MMP, matrix metalloproteinase; AAA, abdominal aortic aneurysm; VC, vehicle treatment plus sham operation control; VA, vehicle treatment plus AAA; QA, quercetin treatment plus AAA; QC, quercetin treatment plus sham operation control.

supplementation of quercetin, a dietary antioxidant with a polyphenolic structure. The antioxidant activity of polyphenols has attracted much attention in relation to their possible role in the prevention of chronic diseases (22). In particular, it was previously reported that resveratrol, another polyphenolic compound, counteracts systemic (23) and local (24) oxidative stress and limits experimental AAA progression. Moreover, a variety of medications and interventions have been proven to successfully suppress experimental aneurysm formation through a ROS-based mechanism (6-8). Thus, it was hypothesized that the aneurysm-inhibitory effect of quercetin in the present study may, in part, associate with its lower oxidation-reduction potential.

Oxidative stress is the result of a redox imbalance between the generation of ROS and the secondary response from the endogenous antioxidant network. Results from the present study indicate a local upregulation of the endogenous antioxidant system, including Mn-SOD and GPxs during $\mathrm{CaCl}_{2}$-induced AAA formation. Mn-SOD and GPxs are key scavengers of ROS, for example, $\mathrm{H}_{2} \mathrm{O}_{2}$ and lipid hydroperoxides $(25,26)$. Therefore, increases in Mn-SOD and GPxs may be a compensatory response for an increase in ROS in 
the mouse aorta following exposure to $\mathrm{CaCl}_{2}$. Quercetin, by restraining ROS levels, prevents the elevation of those antioxidant enzymes, coinciding with other study results (27-29), which have reported the protective effect of quercetin on organ injury.

The enhanced expression of NADPH oxidase, an enzyme that catalyzes the production of $\mathrm{O}_{2}{ }^{--}$from oxygen and $\mathrm{NADPH}$, is a major pathway of ROS formation in the vascular wall (3). Inhibition of ROS production by oral administration of apocynin, a specific inhibitor of NADPH oxidases, attenuates AAA formation in a murine model (8). It was also reported that quercetin prevented the increase in aortic $\mathrm{O}_{2}^{-{ }^{-}}$production through downregulation of $\mathrm{p} 47 \mathrm{phox}$ expression in vivo and in vitro $(14,15)$. Furthermore, Thomas et al (30) have shown that $\mathrm{p} 47$ phox deficiency reduced oxidative stress and markedly attenuated AAA formation. The present study found that quercetin treatment significantly eliminated gene and protein expression of p47phox NADPH oxidase and these data, together, demonstrate that quercetin is able to reduce ROS formation via modulation of the $\mathrm{p} 47$ phox subunit during AAA development.

It is noteworthy that, in a previous study, iNOS deficient mice were partly resistant to aneurysm induction by $\mathrm{CaCl}_{2}$ (8). Nitric oxide synthase (NOS) is also a source of ROS and increased cellular expression of iNOS is specifically associated with large quantities of nitric oxide produced during chronic inflammation (31). The present study found that expression of iNOS in the aortic wall was inhibited following quercetin treatment. It has been reported that under inflammation-mimicking conditions, quercetin may inhibits iNOS expression in cultured monocytes $(32,33)$. This result indicates another mechanism through which quercetin may impact ROS generation.

More importantly, oxidative stress has been reported to activate MMPs $(34,35)$, a family of enzymes with the capacity to cleave several components of the extracellular matrix, including elastin and collagen. It is generally hypothesized that MMPs are putative therapeutic targets in the prevention of AAA (1). Our study group has previously reported that treatment of mice with quercetin prevents aortic wall destruction in the $\mathrm{CaCl}_{2}$-induced AAA model, which is associated with a reduction in the expression of MMP-2 and -9 (16). In the current study, similar results were observed when determining the enzymatic activities of MMPs in vitro by gelatin zymography. MMPs are primarily regulated at the gene transcriptional level by various factors, including cytokines, growth factors, ROS and reactive nitrogen species (RNS). AP-1, a major downstream target of JNK, is an essential transcription factor for MMP expression (36-38). The present study shows that quercetin treatment significantly inhibited AP-1 activation, accompanied by decreased phosphorylation of JNK in AAA tissues. JNK, also known as stress-activated protein kinase, is hypothesized to be involved in a number of cellular stress responses. It is well established that ROS produced from NADPH oxidase and RNS are potent inducers of JNK (39). Moreover, the existing evidence indicates that JNK has an important role in AAA. Yoshimura et al (40) have demonstrated that pharmacological inhibition of JNK reduces MMP levels and prevents the development of AAA. Furthermore, JNK inhibition caused regression of established aneurysm in $\mathrm{CaCl}_{2}$ - and angiotensin II-infusion-induced AAA models. Activation of JNK leads to modulation of other kinases, their nuclear translocation and subsequent phosphorylation of a number of transcription factors, including AP-1 (41). Thus, data from the present study indicated that quercetin reduces oxidative stress and blocks aneurysm formation, which may occur via the mediation of the JNK/AP-1 pathway and MMP modulation.

In conclusion, the present study demonstrated that an antioxidative mechanism is involved in the preventive action of quercetin on $\mathrm{CaCl}_{2}$-induced AAA. This is notable as AAA is a chronic and serious condition for which no medical treatment currently exists. In addition, the compound has been observed to be effective in reducing the risk factors of cardiovascular disease that often occur simultaneously with AAA $(1,9)$. Although it is unclear whether these experimental observations extend to aneurysmal degeneration as it occurs in humans, it is likely to be a point of interest to explore in future investigations.

\section{Acknowledgements}

The present study was supported by grants from the Innovation Projects in Medical Science and Technology of Nanjing Military Region (grant nos. $08 Z 028$ and 10MA090) and the National Natural Science Foundation of China (grant no. 81172032).

\section{References}

1. Sakalihasan N, Limet R and Defawe OD: Abdominal aortic aneurysm. Lancet 365: 1577-1589, 2005.

2. Lederle FA: The natural history of abdominal aortic aneurysm. Acta Chir Belg 109: 7-12, 2009.

3. Keaney JF: Oxidative stress and the vascular wall. Circulation 112: 2585-2588, 2005

4. Marumo T, Schini-Kerth VB, Fisslthaler B and Busse R: Platelet-derived growth factor-stimulated superoxide anion production modulates activation of transcription factor NF-kappaB and expression of monocyte chemoattractant protein 1 in human aortic smooth muscle cells. Circulation 96: 2361-2367, 1997.

5. McCormick ML, Gavrila D and Weintraub NL: Role of oxidative stress in the pathogenesis of abdominal aortic aneurysms. Arterioscler Thromb Vasc Biol 27: 461-469, 2007.

6. Wang M, Lee E, Song W, et al: Microsomal prostaglandin E synthase-1 deletion suppresses oxidative stress and angiotensin II-induced abdominal aortic aneurysm formation. Circulation 117: 1302-1309, 2008.

7. Satoh K, Nigro P, Matoba T, et al: Cyclophilin A enhances vascular oxidative stress and the development of angiotensin II-induced aortic aneurysms. Nat Med 15: 649-656, 2009.

8. Xiong WF, Mactaggart J, Knispel R, et al: Inhibition of reactive oxygen species attenuates aneurysm formation in a murine model. Atherosclerosis 202: 128-134, 2009.

9. Perez-Vizcaino F and Duarte J: Flavonols and cardiovascular disease. Mol Aspects Med 31: 478-494, 2010.

10. Hanasaki Y, Ogawa S and Fukui S: The correlation between active oxygens scavenging and antioxidative effects of flavonoids. Free Radic Biol Med 16: 845-850, 1994.

11. Loke WM, Proudfoot JM, McKinley AJ, et al: Quercetin and its in vivo metabolites inhibit neutrophil-mediated low-density lipoprotein oxidation. J Agric Food Chem 56: 3609-3615, 2008.

12. Loke WM, Proudfoot JM, Hodgson JM, et al: Specific dietary polyphenols attenuate atherosclerosis in apolipoprotein E knockout mice by alleviating inflammation and endothelial dysfunction. Arterioscler Thromb Vasc Biol 30: 749-757, 2010.

13. Golledge $\mathrm{J}$ and Norman PE: Atherosclerosis and abdominal aortic aneurysm: cause, response, or common risk factors? Arterioscler Thromb Vasc Biol 30: 1075-1077, 2010. 
14. Sanchez M, Galisteo M, Vera R, et al: Quercetin downregulates NADPH oxidase, increases eNOS activity and prevents endothelial dysfunction in spontaneously hypertensive rats. J Hypertens 24: 75-84, 2006.

15. Romero M, Jiménez R, Sánchez M, et al: Quercetin inhibits vascular superoxide production induced by endothelin-1: Role of NADPH oxidase, uncoupled eNOS and PKC. Atherosclerosis 202: 58-67, 2009

16. Wang L, Wang B, Li H, et al: Quercetin, a flavonoid with anti-inflammatory activity, suppresses the development of abdominal aortic aneurysms in mice. Eur J Pharmacol 690: 133-141, 2012.

17. Zhao H, Kalivendi S, Zhang H, et al: Superoxide reacts with hydroethidine but forms a fluorescent product that is distinctly different from ethidium: potential implications in intracellular fluorescence detection of superoxide. Free Radic Biol Med 34: 1359-1368, 2003

18. Pryor WA and Squadrito GL: The chemistry of peroxynitrite: a product from the reaction of nitric oxide with superoxide. Am J Physiol 268: L699-L722, 1995.

19. Wang Y, Krishna S and Golledge J: The calcium chloride-induced rodent model of abdominal aortic aneurysm. Atherosclerosis 226 29-39, 2013.

20. Miller FJ Jr, Sharp WJ, Fang X, Oberley LW, Oberley TD and Weintraub NL: Oxidative stress in human abdominal aortic aneurysms: a potential mediator of aneurysmal remodeling. Arterioscler Thromb Vasc Biol 22: 560-565, 2002.

21. Yajima N, Masuda M, Miyazaki M, Nakajima N, Chien S and Shyy JYJ: Oxidative stress is involved in the development of experimental abdominal aortic aneurysm: A study of the transcription profile with complementary DNA microarray. J Vasc Surg 36: 379-385, 2002.

22. Schini-Kerth VB, Etienne-Selloum N, Chataigneau T and Auger C: Vascular protection by natural product-derived polyphenols: in vitro and in vivo evidence. Planta Med 77: 1161-1167, 2011.

23. Palmieri D, Pane B, Barisione C, et al: Resveratrol counteracts systemic and local inflammation involved in early abdominal aortic aneurysm development. J Surg Res 171: e237-e246, 2011.

24. Kaneko H, Anzai T, Morisawa M, et al: Resveratrol prevents the development of abdominal aortic aneurysm through attenuation of inflammation, oxidative stress, and neovascularization. Atherosclerosis 217: 350-357, 2011.

25. Griendling KK and FitzGerald GA: Oxidative stress and cardiovascular injury: Part I: basic mechanisms and in vivo monitoring of ROS. Circulation 108: 1912-1916, 2003.

26. Miyamoto Y, Koh YH, Park YS, et al: Oxidative stress caused by inactivation of glutathione peroxidase and adaptive responses. Biol Chem 384: 567-574, 2003.

27. Dias AS, Porawski M, Alonso M, Marroni N, Collado PS and González-Gallego J: Quercetin decreases oxidative stress, NF-kappaB activation, and iNOS overexpression in liver of streptozotocin-induced diabetic rats. J Nutr 135: 2299-2304, 2005
28. Mahesh T and Menon VP: Quercetin allievates oxidative stress in streptozotocin-induced diabetic rats. Phytother Res 18: 123-127, 2004.

29. Moreira AJ, Fraga C, Alonso M, et al: Quercetin prevents oxidative stress and NF-kappa B activation in gastric mucosa of portal hypertensive rats. Biochem Pharmacol 68: 1939-1946, 2004.

30. Thomas M, Gavrila D, McCormick ML, et al: Deletion of p47(phox) attenuates angiotensin II-induced abdominal aortic aneurysm formation in apolipoprotein E-deficient mice. Circulation 114: 404-413, 2006.

31. Stuehr DJ: Mammalian nitric oxide synthases. Biochim Biophys Acta 1411: 217-230, 1999

32. Kim BH, Cho SM, Reddy AM, Kim YS, Min KR and Kim Y: Down-regulatory effect of quercitrin gallate on nuclear factor-kappaB-dependent inducible nitric oxide synthase expression in lipopolysaccharide-stimulated macrophages RAW 264.7. Biochem Pharmacol 69: 1577-1583, 2005.

33. Bhaskar S, Shalini V and Helen A: Quercetin regulates oxidized LDL induced inflammatory changes in human PBMCs by modulating the TLR-NF-kappaB signaling pathway. Immunobiology 216: 367-373, 2011.

34. Castier Y, Brandes RP, Leseche G, Tedgui A and Lehoux S: p47phox-Dependent NADPH oxidase regulates flow-induced vascular remodeling. Circ Res 97: 533-540, 2005.

35. Ejiri J, Inoue N, Tsukube T, et al: Oxidative stress in the pathogenesis of thoracic aortic aneurysm. Cardiovasc Res 59: 988-996, 2003.

36. Liu X, Manzano G, Lovett DH and Kim HT: Role of AP-1 and RE-1 binding sites in matrix metalloproteinase-2 transcriptional regulation in skeletal muscle atrophy. Biochem Biophys Res Commun 396: 219-223, 2010.

37. Kandasamy AD, Chow AK, Ali MAM and Schulz R: Matrix metalloproteinase-2 and myocardial oxidative stress injury: beyond the matrix. Cardiovasc Res 85: 413-423, 2010.

38. Kar S, Subbaram S, Carrico PM and Melendez JA: Redox-control of matrix metalloproteinase-1: A critical link between free radicals, matrix remodeling and degenerative disease. Respir Physiol Neurobiol 174: 299-306, 2010.

39. Shen HM and Liu ZG: JNK signaling pathway is a key modulator in cell death mediated by reactive oxygen and nitrogen species. Free Radic Biol Med 40: 928-939, 2006.

40. Yoshimura K, Aoki H, Ikeda Y, et al: Regression of abdominal aortic aneurysm by inhibition of c-Jun N-terminal kinase. Nat Med 11: 1330-1338, 2005.

41. Manning AM and Davis RJ: Targeting JNK for therapeutic benefit: from junk to gold? Nat Rev Drug Discov 2: 554-565, 2003. 